\title{
Solution of the Hartree-Fock-Slater equations for diatomic molecules by the finite-element method
}

\author{
D. Heinemann, B. Fricke, and D. Kolb \\ Physics Department, University of Kassel, D-3500 Kassel, West Germany
}

(Received 13 June 1988)

\begin{abstract}
We present the finite-element method in its application to solving quantum-mechanical problems for diatomic molecules. Results for Hartree-Fock calculations of $\mathrm{H}_{2}$ and Hartree-Fock-Slater calculations for molecules like $\mathrm{N}_{2}$ and $\mathrm{CO}$ are presented. The accuracy achieved with fewer than 5000 grid points for the total energies of these systems is $10^{-8}$ a.u., which is about two orders of magnitude better than the accuracy of any other available method.
\end{abstract}

\section{INTRODUCTION}

The finite-element method (FEM) is a well-known technique to solve static and dynamical problems in engineering science. Examples can be found in the textbooks of Refs. 1-3. The idea of this method is to split the space into a number of two or three-dimensional domains and to describe the properties of interest for each of these elements separately. The connection between these elements is done via boundary conditions. The standard approach in engineering science is to use low-order polynomials in order to specify the interesting quantities on each element.

We applied the FEM to quantum-mechanical problems such as the electronic structure of atoms or small diatomic molecules to obtain good wave functions and total energies. Here the accuracy required is very high. In order to achieve this accuracy we had to use high-order polynomials on each element and (in order to minimize the total number of points) a small number of elements.

Section II briefly describes the Hartree-Fock-Slater equations which we are going to solve, whereas Sec. III defines the coordinate system for the diatomic molecules discussed by us. The application of the finite-element method to this problem is discussed in Sec. IV followed by description of the necessary boundary conditions and self-consistency. Finally, we present results for the Hartree-Fock equations of the ground state of $\mathbf{H}_{2}$, and the Hartree-Fock-Slater equations of $\mathrm{N}_{2}$ and $\mathrm{CO}$ as examples. The last section contains the conclusion and outlook.

\section{HARTREE-FOCK-SLATER EQUATIONS}

Many publications ${ }^{4,5}$ describe the Hartree-Fock method and its derivation in detail. We will, therefore, merely provide a very brief description to define the physical approximation and the equations which we are going to solve with the FEM. Using a single Slater determinant wave function for the $N$ electrons in a diatomic molecule,

$\Phi_{\text {tot }}\left(\mathbf{r}_{1}, \mathbf{r}_{2}, \ldots, \mathbf{r}_{N}\right)$

$$
=(N !)^{-1 / 2}\left|\begin{array}{ccc}
\phi_{1}\left(\mathbf{r}_{1}\right) & \cdots & \phi_{1}\left(\mathbf{r}_{N}\right) \\
\vdots & & \vdots \\
\phi_{N}\left(\mathbf{r}_{1}\right) & \cdots & \phi_{N}\left(\mathbf{r}_{N}\right)
\end{array}\right|,
$$

with the one-electron wave functions $\phi_{i}\left(\mathbf{r}_{i}\right)$ and the nonrelativistic Hamiltonian

$$
H=-\frac{1}{2} \sum_{i=1}^{N} \nabla_{i}^{2}-\sum_{i=1}^{N} \sum_{n=1}^{2} \frac{Z_{n}}{\left|\mathbf{r}_{i}-\mathbf{R}_{n}\right|}-\sum_{\substack{i, j=1 \\ i<j}}^{N} \frac{1}{\left|\mathbf{r}_{i}-\mathbf{r}_{j}\right|},
$$

the total energy of such a system can be calculated:

$$
\begin{aligned}
E_{T}= & -\sum_{i=1}^{N} \int \phi_{i}^{*}\left[\frac{1}{2} \nabla_{i}^{2}-\sum_{n=1}^{2} \frac{Z_{n}}{\left|\mathbf{R}_{n}-\mathbf{r}_{i}\right|}\right] \phi_{i} d \mathbf{r}_{i}+\frac{1}{2} \sum_{i, j=1}^{N} \iint \phi_{i}^{*}\left(\mathbf{r}_{i}\right) \phi_{j}^{*}\left(\mathbf{r}_{j}\right) \frac{1}{\left|\mathbf{r}_{i}-\mathbf{r}_{j}\right|} \phi_{i}\left(\mathbf{r}_{i}\right) \phi_{j}\left(\mathbf{r}_{j}\right) d \mathbf{r}_{i} d \mathbf{r}_{j} \\
& -\frac{1}{2} \sum_{i, j=1}^{N} \iint \phi_{i}^{*}\left(\mathbf{r}_{i}\right) \phi_{j}^{*}\left(\mathbf{r}_{j}\right) \frac{1}{\left|\mathbf{r}_{i}-\mathbf{r}_{j}\right|} \phi_{j}\left(\mathbf{r}_{i}\right) \phi_{i}\left(\mathbf{r}_{j}\right) d \mathbf{r}_{i} d \mathbf{r}_{j}
\end{aligned}
$$

The variation of this total energy with respect to the one-electron wave functions $\phi_{i}\left(\mathbf{r}_{i}\right)$, subject to the constraint of their orthonormality, leads to the Hartree-Fock equations

$$
H_{\mathrm{HF}}(\mathbf{r}) \phi_{i}(\mathbf{r})=\varepsilon_{i} \phi_{i}(\mathbf{r}), \quad i=1,2, \ldots, N .
$$

The Hartree-Fock operator $H_{\mathrm{HF}}(\mathbf{r})$ is defined by

$$
H_{\mathrm{HF}}(\mathbf{r})=h(\mathbf{r})+V^{C}(\mathbf{r})+V^{X}(\mathbf{r})
$$

with 


$$
\begin{aligned}
& h(\mathbf{r})=-\frac{1}{2} \nabla^{2}-\frac{Z_{1}}{\left|\mathbf{r}_{1}-\mathbf{r}\right|}-\frac{Z_{2}}{\left|\mathbf{r}_{2}-\mathbf{r}\right|} \\
& V^{C}(\mathbf{r})=\sum_{i=1}^{N} \int \phi_{i}^{*}\left(\mathbf{r}^{\prime}\right) \frac{1}{\left|\mathbf{r}-\mathbf{r}^{\prime}\right|} \phi_{i}\left(\mathbf{r}^{\prime}\right) d \mathbf{r}^{\prime}
\end{aligned}
$$

and

$$
V^{X}(\mathbf{r}) \phi_{j}(\mathbf{r})=\sum_{i=1}^{N} \int \phi_{i}^{*}\left(\mathbf{r}^{\prime}\right) \frac{1}{\left|\mathbf{r}-\mathbf{r}^{\prime}\right|} \phi_{j}\left(\mathbf{r}^{\prime}\right) d \mathbf{r}^{\prime} \phi_{i}(\mathbf{r})
$$

In addition, the interelectronic Coulomb potential $V^{C}(\mathbf{r})$ satisfies the Poisson equation

$$
\nabla^{2} V^{C}(\mathbf{r})=-4 \pi \rho(\mathbf{r})
$$

where $\rho(\mathbf{r})$ is the total electronic density.

In the case of the Hartree-Fock-Slater equations the nonlocal exchange potential $V^{X}(\mathbf{r})$ of the Hartree-Fock equations is replaced by the local statistical exchange potential $^{6-8}$

$$
V^{X}(\mathbf{r})=-\frac{3}{2} \alpha[3 / \pi \rho(\mathbf{r})]^{1 / 3},
$$

with $\alpha$ a constant. The simplified form of a local exchange with $\alpha=0.7$ is used here because the solution of the Hartree-Fock-Slater (HFS) equations with the FEM discussed below depends only on the local form of the approximation. With this approach the total energy of the molecular system is calculated as

$E_{\mathrm{tot}}=\sum_{i=1}^{N} \varepsilon_{i}-\frac{1}{2} \int \rho(\mathbf{r}) V^{C}(\mathbf{r}) d \mathbf{r}-\frac{1}{4} \int \rho(\mathbf{r}) V^{X}(\mathbf{r}) d \mathbf{r}$

The Hartree-Fock-Slater equations (4), with the exchange part according to Eq. (8), are a system of coupled integro-differential equations. The differential equations to be solved are the Schrödinger-type equations derived from Eq. (4) with potentials kept fixed, and the Poisson equation (7) relating the potential $V^{C}$ to the electronic density $\rho$. The solution of the Hartree-Fock-Slater equations (4) has to be achieved iteratively.

\section{CHOICE OF THE COORDINATE SYSTEM}

For a numerical solution of the Hartree-Fock-Slater differential equations for a diatomic molecule the coordinate system must be chosen with great care. Because of the axial symmetry of diatomic molecules we use orthogonal axial-symmetric coordinate systems as they allow a two-dimensional treatment of the problem. They can be written as

$$
\begin{aligned}
& x_{1}=f_{1}(s, t) \cos (\varphi), \\
& x_{2}=f_{1}(s, t) \sin (\varphi), \\
& x_{3}=f_{2}(s, t),
\end{aligned}
$$

where the unknown one-electron wave functions are

$$
\phi_{i}(s, t, \varphi)=\phi_{i}(s, t) \exp (\operatorname{im} \varphi) .
$$

$m$ denotes the projection of the angular momentum onto the internuclear axis. The interelectronic Coulomb potential can be written as follows:

$$
V^{C}(s, t, \varphi)=V^{C}(s, t)
$$

Using the ansatz (10) for the wave functions $\phi_{i}(\mathbf{r})$ the Schrödinger-type equation (4) can be rewritten as a twodimensional differential equation in the axial-symmetric coordinate system

$-\frac{1}{2} \frac{\partial}{\partial s}\left[K_{1}(s, t) \frac{\partial \phi_{i}}{\partial s}\right]-\frac{1}{2} \frac{\partial}{\partial t}\left[K_{2}(s, t) \frac{\partial \phi_{i}}{\partial t}\right]-\frac{1}{2} m^{2} K_{3}(s, t) \phi_{i}(s, t)+K_{4}(s, t) V(s, t) \phi_{i}(s, t)=\varepsilon_{i} K_{4}(s, t) \phi_{i}(s, t)$

In the same way we obtain the two-dimensional differential equation

$$
\begin{array}{r}
-\frac{1}{2} \frac{\partial}{\partial s}\left[K_{1}(s, t) \frac{\partial V^{C}}{\partial s}\right]-\frac{1}{2} \frac{\partial}{\partial t}\left[K_{2}(s, t) \frac{\partial V^{C}}{\partial t}\right] \\
=-4 \pi K_{4}(s, t) \rho(s, t)
\end{array}
$$

for the Poisson equation (7). The coefficient functions $K_{1}, K_{2}, K_{3}$, and $K_{4}$ are calculated from the transformation functions $f_{1}$ and $f_{2}$ through

$$
\begin{aligned}
& K_{1}=g^{1 / 2} / g_{11}, \quad K_{2}=g^{1 / 2} / g_{22}, \\
& K_{3}=g^{1 / 2} / g_{33}, \quad K_{4}=g^{1 / 2},
\end{aligned}
$$

with

$$
g=g_{11} g_{22} g_{33}
$$

and

$$
\begin{aligned}
& g_{11}=\left(\frac{\partial f_{1}}{\partial s}\right)^{2}+\left[\frac{\partial f_{2}}{\partial s}\right]^{2} \\
& g_{22}=\left[\frac{\partial f_{1}}{\partial t}\right]^{2}+\left[\frac{\partial f_{2}}{\partial t}\right]^{2}, \\
& g_{33}=f_{1}^{2}
\end{aligned}
$$

We agree with Laaksonen et al. ${ }^{9-11}$ and Becke ${ }^{12}$ in finding the prolate-spheroidal coordinates most suitable for the properties of the self-consistent calculation of diatomic molecules. The transformation equations for this coordinate system are given by the equations

$$
\begin{aligned}
& x_{1}=(R / 2) \sinh s \sin t \cos \varphi, \\
& x_{2}=(R / 2) \sinh s \sin t \sin \varphi, \\
& x_{3}=(R / 2) \cosh s \cos t .
\end{aligned}
$$

With these transformation equations we can calculate the coefficient functions to be 


$$
\begin{aligned}
& K_{1}(s, t)=K_{2}(s, t)=(R / 2) \sinh s \sin t, \\
& K_{3}(s, t)=(R / 2)\left(\sinh ^{2} s+\sin ^{2} t\right) /(\sinh s \sin t), \\
& K_{4}(s, t)=\left(R^{3} / 2\right)\left(\sinh ^{2} s+\sin ^{2} t\right) \cdot \sinh s \sin t .
\end{aligned}
$$

Other coordinate systems were also tested. For the case of $\mathrm{H}_{2}{ }^{+}$we found the coordinates with the transformation equations

$$
\begin{aligned}
& x_{1}=(R / 2)\left[(1-c \ln s)^{2}-1\right]\left(1-t^{2}\right) \cos \varphi, \\
& x_{2}=(R / 2)\left[(1-c \ln s)^{2}-1\right]\left(1-t^{2}\right) \sin \varphi, \\
& x_{3}=(R / 2)(1-c \ln s) t,
\end{aligned}
$$

to be most useful. By varying the transformation parameter $c$ of the transformation equations (17) very accurate results could be achieved with a very small number of points. ${ }^{13}$ However, for the $\mathrm{H}_{2}$ problem we used the coordinate system defined by the transformation equations

$$
\begin{aligned}
& x_{1}=(R / 2)\left[(\sinh s+1)^{2}-1\right]\left(1-t^{2}\right) \cos \varphi, \\
& x_{2}=(R / 2)\left[(\sinh s+1)^{2}-1\right]\left(1-t^{2}\right) \sin \varphi, \\
& x_{3}=(R / 2)(\sinh s+1) t .
\end{aligned}
$$

The reason for this was that the solution of the Poisson equation within the coordinate system (17) proved to be unstable.

\section{FINITE-ELEMENT METHOD (REFS. 1-3)}

Originally the FEM was developed in engineering science in order to calculate static and dynamic stresses of complicated constructions. Recently, it has been demonstrated ${ }^{14-20}$ that this method can also be used with great success to solve quantum-mechanical problems. In order to apply the FEM to the solution of the Hartree-FockSlater equations, one best starts from the variational equivalent of the second-order partial differential equations which, for the Schrödinger-type equation (12), takes the form

$$
\begin{aligned}
I_{1}=\iint & {\left[-\frac{1}{4} K_{1}\left(\frac{\partial \phi_{i}}{\partial s}\right)^{2}-\frac{1}{4} K_{2}\left(\frac{\partial \phi_{i}}{\partial t}\right)^{2}-\frac{1}{4} m^{2} K_{3}\left(\phi_{i}\right)^{2}\right.} \\
& \left.-\frac{1}{2}\left(V-\varepsilon_{i}\right) K_{4}\left(\phi_{i}\right)^{2}\right] d s d t
\end{aligned}
$$

and for the Poisson equation (13),

$$
\begin{aligned}
& I_{2}=\iint\left[-\frac{1}{2} K_{1}\left(\frac{\partial V^{C}}{\partial s}\right]^{2}-\frac{1}{4} K_{2}\left[\frac{\partial V^{C}}{\partial t}\right]^{2}\right. \\
& \left.+4 \pi K_{4}(s, t) \rho(s, t) V^{\mathrm{c}}\right] d s d t .
\end{aligned}
$$

The idea of the FEM is to subdivide the space into $N_{s}$ small domains called elements. In our case triangles are used to subdivide the two-dimensional space of our problem. And it is at this point that one main advantage of the FEM in contrast to other numerical methods, such as the finite-difference method used by Laaksonen et al., ${ }^{9-11,21,22}$ becomes important. The size and shape of elements can be defined very freely so that physical prop- erties can be taken into account, e.g., one can use small elements in regions of physical importance and large elements in regions of lesser weight. Thus the point distribution can be adapted to a given problem.

The next step in the FEM is to choose a trial function for the element to approximate the solution of the differential equation. In order to fulfill the continuity criterion of the solution from one element to the next, the trial functions are written in a special way. For each element a number of points, the nodal points, are chosen. If $u^{e}(s, t)$ is the approximation of the unknown function over the element with the number $e$, this function is written as a linear combination of the nodal values

$$
u^{e}(s, t)=\sum_{i=1}^{p} u_{i}^{e} N_{i}^{e}(s, t)
$$

where $u_{i}^{e}$ is the $i$ th nodal value of the eth element. The function $N_{i}^{e}(s, t)$ is the $i$ th shape function. The sum runs over all nodal values of the element. Two main types of elements exist, namely, the Lagrangian- and the Hermitian-type elements. (If the interpolation of an unknown function is calculated only due to the values of the function at specified points this is called a Lagrange interpolation. The interpolation of the unknown function which takes also the derivatives of the function into account is called a Hermite interpolation. ${ }^{23}$ ) Lagrangian elements have only one nodal value per nodal point, namely, the value of the unknown function. Hermitian elements have more than one nodal value per nodal point, because here also the derivatives of the functions are nodal values. One conclusion of Eq. (21) is that each shape function equals 1 only at a single nodal point of the element and equals 0 at all others. This restriction allows the construction of the shape functions from the given trial function.

We use Lagrange elements with two-dimensional polynomials up to order 6 as trial function. To be able to construct the shape functions we choose as many nodal points for each elements as there are free coefficients of the two-dimensional polynomials. Figure 1 shows the nodal points of an element for a fifth-order polynomial.

Because of the subdivision of the region into $M$ elements the functionals $I_{1}$ [Eq. (19)] and $I_{2}$ [Eq. (20)] can

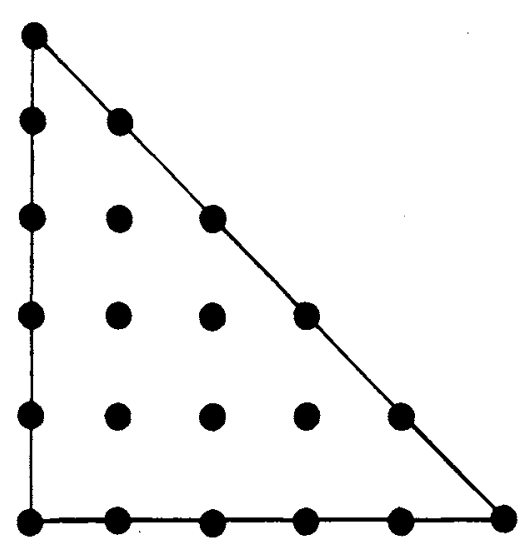

FIG. 1. Nodal points of a fifth-order Lagrangian element. 
be written as a sum of element integrals

$$
I_{1}=\sum_{e=1}^{M} I_{1}^{e}, \quad I_{2}=\sum_{e=1}^{M} I_{2}^{e},
$$

with integrals running over the array of the element $e$.

The substitution of the trial function (21) into the element integrals (22) leads to the following linear expression for the Schrödinger-type equations:

$$
I_{1}^{e}=\left(\mathbf{u}^{e}\right)^{t} \cdot \underline{H}^{e} \cdot \mathbf{u}^{e}-\varepsilon\left(\mathbf{u}^{e}\right)^{t} \cdot \underline{\underline{S}}^{e} \cdot \mathbf{u}^{e},
$$

with the matrix elements

$$
\begin{aligned}
H_{i j}=-\frac{1}{4} \iint & {\left[K_{1} \frac{\partial N_{i}^{e}}{\partial s} \frac{\partial N_{j}^{e}}{\partial s}+K_{2} \frac{\partial N_{i}^{e}}{\partial t} \frac{\partial N_{j}^{e}}{\partial t}\right.} \\
& \left.+\left(K_{3} m^{2}+2 K_{4} V\right) N_{i}^{e} N_{j}^{e}\right] d s d t
\end{aligned}
$$

and

$$
S_{i j}=\iint \frac{1}{2} K_{4} N_{i}^{e} N_{j}^{e} d s d t
$$

For the Poisson equation we obtain the similar matrix expression

$$
I_{2}^{e}=\left(\mathbf{u}_{e}\right)^{t} \cdot \underline{D^{e}} \cdot \mathbf{u}^{e}-\left(\mathbf{u}^{e}\right)^{t} \cdot \mathbf{d}^{e},
$$

with the matrix elements

$D_{i j}=\frac{1}{2} \iint\left[K_{1} \frac{\partial N_{i}^{e}}{\partial s} \frac{\partial N_{j}^{e}}{\partial s}+K_{2} \frac{\partial N_{i}^{e}}{\partial t} \frac{\partial N_{j}^{e}}{\partial t}\right] d s d t$

and the vector elements

$$
d_{i}=4 \pi \iint K_{4} \rho(s, t) N_{i}^{e} d s d t .
$$

The vector $\mathbf{u}^{e}$ is the element nodal vector of the eth element.

Adding the contributions of all elements leads to the expressions

$$
I_{1}=\mathbf{u}^{t} \cdot \underline{\mathrm{H}} \cdot \mathbf{u}-\varepsilon \mathbf{u}^{t} \cdot \underline{\mathbf{S}} \cdot \mathbf{u}
$$

and

$$
I_{2}=\mathbf{u}^{t} \cdot \underline{\mathrm{D}} \cdot \mathbf{u}-\mathbf{u}^{t} \cdot \mathbf{d},
$$

where $\mathbf{u}$ denotes the global nodal vector.

The global matrices $\underline{\mathbf{H}}, \underline{\mathbf{S}}$, and $\underline{\mathrm{D}}$ are band-structured matrices, because for any given nodal variable $u_{i}$ at point $\left(s_{i}, t_{i}\right)$ the sum over all elements runs only over those elements which share this point. As an example, Fig. 2 shows the structure of the global matrix for the subdivision of a rectangular region given in Fig. 3. The nodal points are given by the vertices of the triangles. The nodal variables are numbered from 1 to 6 and the elements from 1 to 4 . The matrix elements of element $1(2,3,4)$ are marked with the symbols $O(X, \square, \triangle)$.

With the substitution of the trial functions the functionals $I_{1}$ and $I_{2}$ are seen to be functions of the nodal variables $u_{i}$. The conditions for $I_{1}$ and $I_{2}$ to be minimal are

$$
\frac{\partial I_{1}}{\partial u_{i}}=0, \quad \frac{\partial I_{2}}{\partial u_{i}}=0, \quad i=1, \ldots, N_{p} .
$$

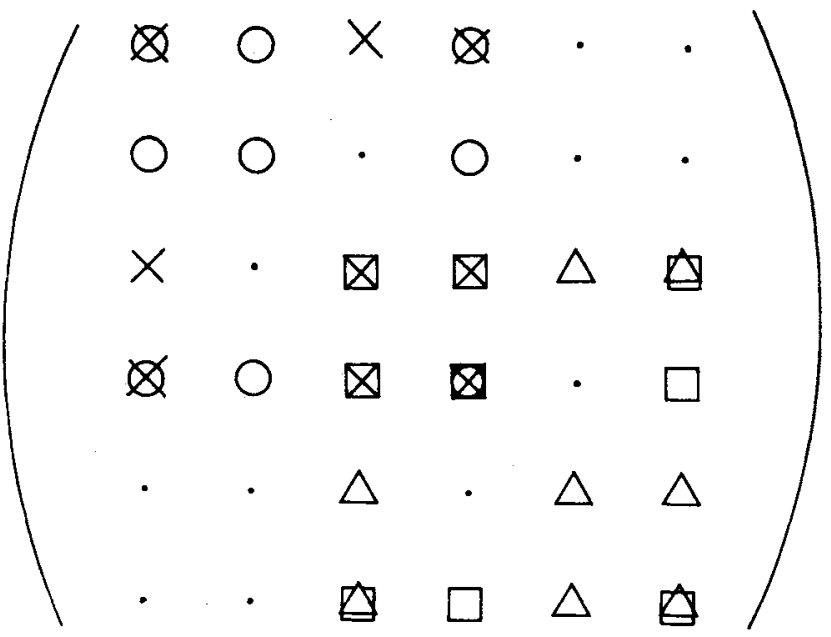

FIG. 2. Structure of the global matrices resulting from the subdivision of a rectangular region given in Fig. 3. The matrix elements of element $1(2,3,4)$ are symbolized by $\circ(X, \square, \triangle)$.

These conditions lead to the matrix eigenvalue problem

$$
\underline{\mathbf{H}} \cdot \mathbf{u}=\boldsymbol{\varepsilon} \cdot \underline{\mathbf{S}} \cdot \mathbf{u}
$$

for the Schrödinger-type equation and to the matrix equation

$$
\underline{D} \cdot \mathbf{u}=\mathbf{d}
$$

for the Poisson equation. The order of this matrix equation is equal to the number $N_{p}$ of nodal variables. For the Lagrangian elements used by us, this is equal to the total number of points.

The calculation of the matrix elements $(24,25,27,38)$ is done numerically with a conical product Gauss integration rule. ${ }^{24}$ A number of only $7 \times 7$ integration points for each element proved to be sufficient for all calculations.

The matrix eigenvalue problem is solved by an inverse vector iteration method ${ }^{3,25}$ with modifications due to the iterative solution of the Hartree-Fock-Slater equations. The matrix equation is solved by a Cholesky decomposition. ${ }^{3,26}$ All algorithms used at this point take care of the band structure of the global matrices. ${ }^{3}$

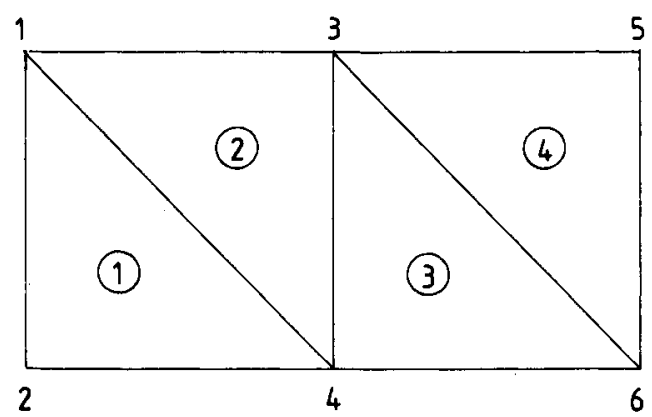

FIG. 3. Subdivision of a rectangular region with four triangular elements. The enumeration of the nodal points leads to a bandwidth of 3 for the corresponding global matrices. 


\section{BOUNDARY CONDITIONS}

To obtain the boundary conditions for the unknown wave functions and the unknown potential it is useful to introduce the coordinates

$$
\begin{aligned}
& \xi=\left(r_{1}+r_{2}\right) / R, \\
& \eta=\left(r_{1}-r_{2}\right) / R,
\end{aligned}
$$

where the relation to the coordinates $s$ and $t$ used above (15) is given by $\xi=$ coshs and $\eta=$ cost. Within these coordinates the asymptotic behavior ${ }^{11}$ for the wave functions $\phi(\xi, \eta)$ is

$$
\phi \simeq \exp \left[-(-2 \varepsilon)^{1 / 2}(R / 2) \xi\right], \quad \xi \rightarrow \infty
$$

where $\varepsilon$ is the one-electron energy eigenvalue. For the boundary conditions of the wave functions we use the value $\phi=0$ for practical infinity. The error of this value can be checked either from Eq. (35) or by variation of the practical infinity point.

The asymptotic behavior of the interelectronic potential $V^{C}$ is

$$
V^{C} \simeq N /(R / 2 \xi)=N /[(R / 2) \cosh s], \quad \xi \rightarrow \infty .
$$

To remove the $N / \xi$ decrease of the potential $V^{C}$ we in-

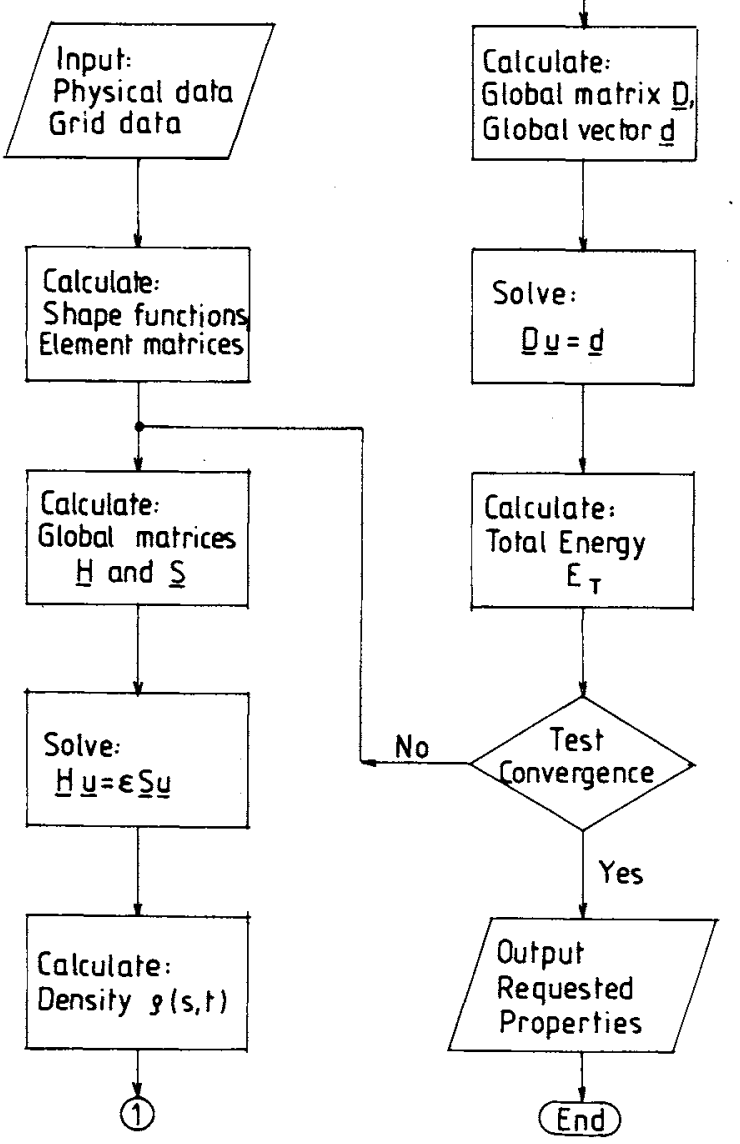

FIG. 4. Flowchart of the finite-element program solving the Hartree-Fock-Slater equations.

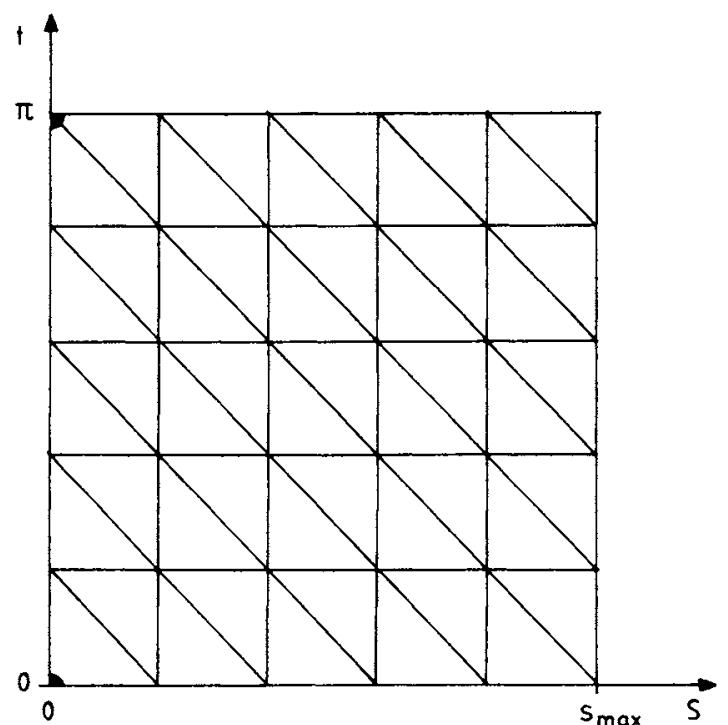

FIG. 5. Equidistant subdivision of the $(s, t)$ region with 50 triangular elements. The nodal points on one element are distributed according to Fig. 1 .

troduce a function $g(s, t)$. Thus

$$
V^{C}=g(s, t) f^{V}(s, t) .
$$

The function $f^{V}(s, t)$ is solved by the FEM. The function $g(s, t)$ is chosen to be

$$
g(s, t)=N /[(R / 2) \cosh s] .
$$

The resulting boundary condition for $f^{V}(s, t)$ is

$$
f^{V}(s, t)=1
$$

at practical infinity. Other choices for the function $g(s, t)$ are possible. Favorable are functions which also approximate the potential for small $\xi$ and include the dipole potential of the electronic density for large $\xi$.

\section{SELF-CONSISTENT SOLUTION}

Figure 4 shows the flow diagram of the computer program. As a convergence criterion we use three parameters. First the change $\Delta_{e}$ of the one-electron energy eigenvalues, second the maximal change $\Delta_{V}$ of the interelectronic potential, and third the change $\Delta_{E}$ of the total energy. The most accurate results were obtained with

TABLE I. Convergence properties of total energy and energy eigenvalue of the system $\mathrm{H}_{2}$ with increasing number of points. All values are given in a.u.

\begin{tabular}{ccc}
\hline \hline & $\mathrm{H}_{2}$, internuclear distance $R=1.40$ a.u. \\
Points & $E_{T}$ & $\varepsilon$ \\
\hline $6 \times 6$ & -1.12873769 & -0.59583041 \\
$11 \times 11$ & -1.13362024 & -0.59466119 \\
$16 \times 11$ & -1.13362884 & -0.59465813 \\
$21 \times 11$ & -1.13362949 & -0.59465860 \\
$26 \times 11$ & -1.13362956 & -0.59465856 \\
$41 \times 16$ & $-1.1336295717(2)$ & $-0.5946585694(3)$ \\
\hline
\end{tabular}


TABLE II. Total energy and energy eigenvalues of the system $\mathrm{N}_{2}$ for different grid sizes and different orders of the polynomials over the elements. The last figure for the largest grids is uncertain. All values are given in a.u.

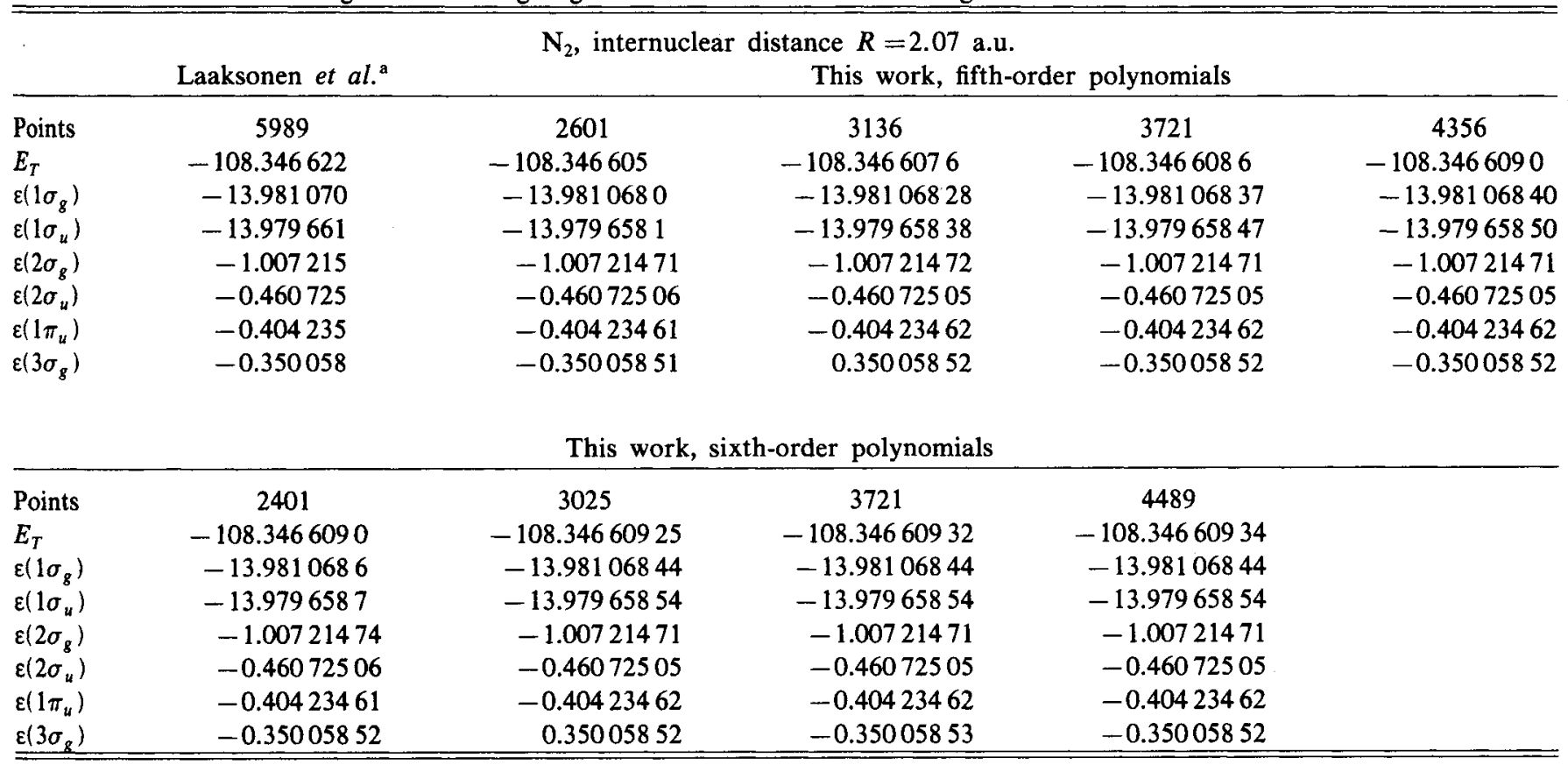

${ }^{\mathrm{a}}$ Reference 11 .

$\Delta_{e}$ and $\Delta_{E}$ set to $10^{-8}$ a.u. and $\Delta_{V}=10^{-8}$. Typically the change of the total energy $\Delta_{E}$ was the most restricting criterion for the convergence.

The self-consistent calculation is initialized by the variable screening potential of Eichler and Wille. ${ }^{27}$ With this starting potential about 20 iterations were needed to achieve convergence to $10^{-8}$.

\section{RESULTS}

We present the results for some of the systems calculated by us. The systems here were chosen to be identical to the systems already calculated by Laaksonen et al., $9-11,22$ in order to compare the numerical quality of the results.

For the simplicity the points were distributed equidistantly in the selected coordinate system. As an example, Fig. 5 shows a triangularization of the two-dimensional region with a total number of 50 elements. For the fifth- order polynomials as trial functions this results in a total number of $26 \times 26$ grid points, and in $31 \times 31$ grid points for the sixth-order polynomials. Such an equidistant point distribution is not at all optimal, and it will be shown that point distributions, which are physically more adequate, will increase the accuracy.

Table I shows the convergence of the total energy and the $1 \sigma_{g}$ level of the system $\mathrm{H}_{2}$ with increasing grid size. For a number of only $26 \times 11$ grid points an accuracy better than $10^{-8}$ was reached with fifth-order polynomials. Increasing the number of grid points by a factor of about 3 to $41 \times 16$ points enabled us to add two more significant digits to a new benchmark with ten-figure accuracy. This shows the stability of the FEM even for highly accurate calculations.

The next system was $\mathbf{N}_{2}$. In Table II we compare the convergence of the results with increasing grid size for both fifth- and for sixth-order polynomials. For 2401

TABLE III. Total energy and energy eigenvalues of the system $\mathrm{CO}$ and $\mathrm{BH}$ for the largest grids used so far. The last figure given is uncertain. All values are given in a.u.

\begin{tabular}{lcccc}
\hline & \multicolumn{2}{c}{ Laaksonen et $^{2}$. $^{\mathrm{a}}$} & \multicolumn{2}{c}{ This work, sixth order } \\
& $\mathrm{BH}$ & $\mathrm{CO}$ & $\mathrm{BH}$ & CO \\
\hline Points & & & 4356 & 4356 \\
$R$ & 2.366 & 2.13 & 2.366 & 2.13 \\
$E_{T}$ & -24.808852 & -112.129925 & -24.80885148 & -112.12991528 \\
$\varepsilon(1 \sigma)$ & -6.5323604 & -18.744146 & -6.53236004 & -18.74414325 \\
$\varepsilon(2 \sigma)$ & -0.4078652 & -9.911347 & -0.40786519 & -9.91134609 \\
$\varepsilon(3 \sigma)$ & -0.1731323 & -1.044171 & 0.17313242 & -1.04417077 \\
$\varepsilon(4 \sigma)$ & & -0.489071 & & -0.48907075 \\
$\varepsilon(1 \pi)$ & & -0.413613 & & -0.41261271 \\
$\varepsilon(5 \sigma)$ & & -0.303029 & & -0.30302991 \\
\hline \hline
\end{tabular}

${ }^{\text {a }}$ Reference 11 . 
TABLE IV. Total energy and energy eigenvalues of the system $\mathrm{N}_{2}$ for two different point distributions. Mesh $A$ refers to an equidistant grid with all elements of equal size, whereas mesh $B$ refers to a logarithmic point distribution in the $s$ coordinate. All values are given in a.u.

\begin{tabular}{lcc}
\hline \hline & $\begin{array}{c}\mathrm{N}_{2}, \text { internuclear distance } R=2.07 \\
\text { Sixth-order polynomials } \\
\text { Mesh } A\end{array}$ & Mesh $B$ \\
\hline Points & 961 & 961 \\
$E_{T}$ & -108.34645 & -108.34659 \\
$\varepsilon\left(1 \sigma_{g}\right)$ & -13.98104 & -13.981066 \\
$\varepsilon\left(1 \sigma_{u}\right)$ & -13.97963 & -13.979656 \\
$\varepsilon\left(2 \sigma_{g}\right)$ & -1.0072143 & -1.0072150 \\
$\varepsilon\left(2 \sigma_{u}\right)$ & -0.4607255 & -0.4607253 \\
$\varepsilon\left(1 \pi_{u}\right)$ & -0.4042346 & -0.4042347 \\
$\varepsilon\left(3 \sigma_{g}\right)$ & -0.3500577 & 0.3500587 \\
\hline \hline
\end{tabular}

points the accuracy of the sixth-order polynomial grid is comparable to that of the 4356-point fifth-order polynomial grid. This comparison shows clearly the advantage of high-order trial functions for the FEM in order to apply this method to quantum-mechanical problems. These results are expected to be accurate within $10^{-8}$ a.u. The results for the asymmetric molecules $\mathrm{CO}$ and $\mathrm{BH}$, given in Table III, are the results obtained with the largest sixth-order grid.

In Table IV a step towards an optimized point distribution is documented. In this table we compare the results of the system $\mathrm{N}_{2}$ for a $31 \times 31$ point grid of sixth order. In mesh $A$ we use an equidistant distribution of the points, whereas in mesh $B$ the $s$ coordinates [Eq. (15)] of the vertices of the triangles are distributed logarithmically. The points used for the $s$ coordinates are about 0.0 , $0.091,0.219,0.348,0.649$, and 1.0 times the maximal $s$ value (for practical infinity we use 25 a.u. in this case). The other nodal points for a single element are distributed equidistantly within that element. With this distribution of elements still far from being optimal the accuracy of the results is about $1.0 \times 10^{-5}$ a.u. This is about a factor of 15 better than the accuracy of $1.5 \times 10^{-4}$ a.u. achieved with the linear mesh $A$.

All calculations were performed on an IBM 3090-40E mainframe. The FORTRAN vectorize option was used for all routines handling the global matrices and vectors, but no special vector routines were used. About $90 \%$ of the CPU time was needed to solve the matrix eigenvalue problem (32) and the matrix equation (33). The CPU time per iteration is about 20 seconds for the $31 \times 31$ point grid with sixth-order polynomials. The increase of CPU time with grid size is about

$$
T_{\mathrm{CPU}} \sim n m_{b}^{2} n_{1} .
$$

Here $n$ denotes the number of grid points, $n_{1}$ is the number of levels to be calculated, and $m b$ is the bandwidth of the global matrices. For the bandwidth $m b$ we can write

$$
m_{b} \sim n^{1 / 2} n_{\text {ord }}
$$

when $n_{\text {ord }}$ is the order of the polynomials of the trial function. The last formula is only true for the regular grids used up to now.

\section{CONCLUSION AND OUTLOOK}

It has been demonstrated that the finite-element method is adequate for solving the two-dimensional Hartree-Fock-Slater equations with high numerical accuracy. The results presented are by two orders of magnitude more accurate than the results achieved by Laaksonen et al. ${ }^{9-11,22}$ with the finite-difference method, although we used a much smaller number of points. Up to now no numerical instabilities have been found in the calculations with increasing grid sizes. The main difference between the engineering approaches of the FEM and the current approach is the need of very-high-order trial functions.

A Hartree-Fock approach to diatomic molecules is one of our next goals. The first attempt towards an optimized element distribution shows a very nice increase in accuracy. With better adapted element distributions we hope to reach very accurate results with a very small number of grid points. If this can be achieved one can think of attacking a three-dimensional numerical calculation within reasonable computer times.

\section{ACKNOWLEDGMENTS}

This work was partially supported by the Deutsche Forschungsgemeinschaft (DFG) and the Gesellschaft für Schwerionenforschung Darmstadt (GSI).
${ }^{1} \mathrm{G}$. Strang and G. Fix, An Analysis of the Finite Element Method (Prentice-Hall, Englewood Cliffs, 1973).

${ }^{2}$ D. H. Norrie and G. de Vries, An Introduction to Finite Element Analysis (Academic, New York, 1978).

${ }^{3}$ H. R. Schwarz, Methode der Finiten Elemente (Teubner, Stuttgart, 1980).

${ }^{4}$ J. C. Slater, Quantum Theory of Atomic Structure, (McGrawHill, New York, 1960).

${ }^{5} \mathrm{C}$. Froese-Fischer, The Hartree-Fock Method for Atoms (McGraw-Hill, New York, 1977).

${ }^{6} \mathrm{~J}$. C. Slater, Phys. Rev. 81, 385 (1951).
${ }^{7}$ R. Gaspar, Acta Phys. Acad. Sci. Hung. 3, 263 (1954).

${ }^{8}$ W. Kohn and L. J. Sham, Phys. Rev. 140, A1133 (1965).

${ }^{9}$ L. Laaksonen, D. Sundholm, and P. Pyykkö, Chem. Phys. Lett. 96, 1 (1983).

${ }^{10}$ L. Laaksonen, D. Sundholm, and P. Pyykkö, Int. J. Quantum Chem. 27, 601 (1985).

${ }^{11}$ L. Laaksonen, D. Sundholm, and P. Pyykkö, Comp. Phys. Rep. 4, 313 (1986).

${ }^{12}$ A. D. Becke, J. Chem. Phys. 76, 6037 (1982).

${ }^{13}$ W. Schulze and D. Kolb, Chem. Phys. Lett. 122, 271 (1985).

${ }^{14}$ A. Askar, A. Cakmak, and H. Rabits, Chem. Phys. 33, 267 
(1978).

${ }^{15}$ M. Duff, H. Rabits, A. Askar, A. Cakmak, and M. Ablowitz, J. Chem. Phys. 72, 1543 (1980).

${ }^{16}$ M. S. Kaschiev, S. I. Vinitsky, and F. R. Vukajlovic, Phys. Rev. A 22, 557 (1980).

${ }^{17}$ W. K. Ford and F. S. Levin, Phys. Rev. A 29, 43 (1984).

${ }^{18} \mathrm{M}$. Mishra, J. Linderberg, and Y. Öhrn, Chem. Phys. Lett. 111, 439 (1984).

${ }^{19}$ F. S. Levin and J. Shertzer, Phys. Rev. A 32, 3285 (1985).

${ }^{20} \mathrm{R}$. Jaquet, Theor. Chim. Acta 71, 425 (1987).

${ }^{21}$ L. Laaksonen, P. Pyykkö, and D. Sundholm, Int. J. Quantum
Chem. 23, 309 (1983).

${ }^{22}$ L. Laaksonen, P. Pyykkö, and D. Sundholm, Int. J. Quantum Chem. 23, 319 (1983).

${ }^{23}$ K. E. Atkinson, An Introduction to Numerical Analysis (Wiley, New York, 1978).

${ }^{24}$ A. H. Stroud, Approximate Calculation of Multiple Integrals (Prentice-Hall, Englewood Cliffs, 1971).

${ }^{25}$ R. Gruber, Comput. Phys. Commun. 10, 30 (1975).

${ }^{26} \mathrm{~J}$. H. Wilkonson and C. Reinsch, Linear Algebra (Springer, New York, 1971).

${ }^{27}$ J. Eichler and U. Wille, Phys. Rev. Lett. 33, 56 (1974). 